\title{
Editorial
}

\section{Local anaesthesia for cataract surgery}

I have always believed that general anaesthesia provides for the surgeon and the patient a wider margin of safety during intraocular surgery than is possible with local anaesthesia. This is because the anaesthetist is able to control the choroidal blood flow with positive pressure hyperventilation during the surgical procedure, and hence maintain a desirably soft and still eye. Despite this belief, which is widely held, general anaesthesia is increasingly regarded as an unaffordable luxury in ophthalmology, particularly for cataract surgery.

The surge in popularity of local anaesthesia for cataract surgery does not appear to have been associated with a significant rise in complications during or after the operation, though hard data are hard to come by. It even appears to be possible to achieve excellent results with less and less local anaesthetic agent and without recourse to retrobulbar or even peribulbar injection. Techniques are described which seem to be designed to produce adequate peroperative analgesia with only a nod in the direction of ocular akinesia, other than by securing the eye with a superior rectus suture.

This issue sees the publication of work by Redmond and Dallas and by RJH Smith, both papers dealing with the subject of local anaesthesia for cataract surgery. Both studies, though limited in their scope, seem to indicate that the greatly abbreviated dosage and technique should find favour. I have tried each of the described methods on an occasional and ad hoc basis without serious mishap. I could not, however, compare favourably these experiences with the security and peace of mind I enjoy with the help of an anaesthetist colleague.

Many factors are forcing us to change our ways. Socioeconomic concerns promote more day case surgery, sometimes with inadequate consideration for the patients's welfare. Patients are not universally keen on the idea of going home immediately after surgery (often a journey of many miles for hospitals in London) when they have to return again for inspection next day. In the United States, where this process is the norm, hotels and motels have sprung up adjacent to the busy cataract facilities and patients are prepared and expect to pay for the use of them. Such enterprise does not exist in the UK, and NHS patients, I venture to suggest, would be unwilling to pay were it to emerge.

The fact remains that we do not know what is best. In their summary Redmond and Dallas state that their results may encourage investigation, and this is certainly needed. Only evidence from a prospective and preferably randomised study will serve to provide an answer. One should also not forget that day case surgery no longer requires local anaesthesia only. Agents and techniques for general anaesthesia are changing fast, so that rapid and safe recovery is becoming a realistic expectation. Thus may we be able to have our cake and eat it. 